\title{
BANANA at WNUT-2020 Task 2: Identifying COVID-19 Information on Twitter by Combining Deep Learning and Transfer Learning Models
}

\author{
Tin Van Huynh \\ University of Information Technology \\ VNU-HCM, Vietnam \\ 16521827@gm.uit.edu.vn
}

\author{
Luan Thanh Nguyen \\ University of Information Technology \\ VNU-HCM, Vietnam \\ 17520721@gm.uit.edu.vn
}

\author{
Son T. Luu \\ University of Information Technology \\ VNU-HCM, Vietnam \\ sonlteuit.edu.vn
}

\begin{abstract}
The outbreak COVID-19 virus caused a significant impact on the health of people all over the world. Therefore, it is essential to have a piece of constant and accurate information about the disease with everyone. This paper describes our prediction system for WNUT-2020 Task 2: Identification of Informative COVID-19 English Tweets. The dataset for this task contains size 10,000 tweets in English labeled by humans. The ensemble model from our three transformer and deep learning models is used for the final prediction. The experimental result indicates that we have achieved $\mathrm{F} 1$ for the INFORMATIVE label on our systems at $88.81 \%$ on the test set.
\end{abstract}

\section{Introduction}

The rapid spread of the coronavirus (COVID-19) has caused a global health crisis. This virus is hazardous to people's health and causes a big panic all over the world. Statistics show that each day there are 4 million tweets related to COVID-19 on Twitter (Lamsal, 2020). Therefore, it is essential to keep track of the information associated with this disease. Along with the development of many social networking platforms such as Twitter and Facebook. This is the primary way that helps people capture information about COVID-19 regularly. However, there is much content appearing daily on these social media platforms. Most of them do not have information about the status of COVID-19, such as the number of suspected cases or cases near the user's area.

In this article, we present our approach at WNUT-2020 Task 2 (Nguyen et al., 2020) to identify Tweets containing information about COVID19 on the social networking platform Twitter or not. A Tweet is believed to have information if it includes information such as recovered, suspected, confirmed, and death cases and location or travel

\begin{tabular}{lc}
\hline Tweet & Label \\
\hline A New Rochelle rabbi and a White & 0 \\
Plains doctor are among the 18 con- & \\
firmed coronavirus cases in Westchester. & \\
HTTPURL & \\
\hline Day 5: On a family bike ride to pick & 1 \\
up dinner at @USER Broadway, we & \\
encountered our pre-COVID-19 Land \\
Park happy hour crew keeping up the \\
tradition at an appropriate \#SocialDis- \\
tance.HTTPURL
\end{tabular}

Table 1: Several examples in the WNUT-2020 Task 2 dataset. 0 and 1 stand for INFORMATIVE and UNINFORMATIVE, respectively.

history of the patients. Specifically, we described the problem as follows.

- Input: Given English Tweets on the social networking platform.

- Output: One of two labels (INFORMATIVE and UNINFORMATIVE) predicted by our system.

Several examples are shown in Table 1

In this paper, we have two main contributions as follows.

- Firstly, we implemented four different models based on neural networks and transformers such as Bi-GRU-CNN, BERT, RoBERTa, XLNet to solve the WNUT-2020 Task 2: Identification of informative COVID-19 English Tweets.

- Secondly, we propose a simple ensemble model by combining multiple deep learning and transformer models. This model gives the highest performance compared with the single 
models with F1 on the test set is $88.81 \%$ and on the development set is $90.65 \%$.

\section{Related work}

During the happening of the COVID-19 pandemic, the information about the number of infected cases, the number of patients is vital for governments. Dong et al. (2020) constructed a real-time database for tracking the COVID-19 around the world. This dataset is collected by experts from the World Health Organization (WHO), US CDC, and other medical agencies worldwide and is operated by John Hopkins University. Also, there are many other COVID-19 datasets such as multilingual data collected on Twitter from January 2020 (Chen et al., 2020) or Real World Worry Dataset (RWWD) (Kleinberg et al., 2020).

Besides, on social media, the spreading of COVID-19 information is extremely fast and enormous and sometimes leads to misinformation. Shahi et al. (2020) conducted a pilot study about detecting misinformation about COVID-19 on Twitter by analyzing tweets using standard social media analytics techniques. From the researching results, the authors want to help authorities and social media users counter misinformation. Moreover, the rumors and conspiracy theories within the emergence times of COVID-19 spreading had made communities feel fearmongering and panicky, which lead to racism about COVID-19 patients and citizens from infected countries, and mass purchase of face masks as well as the shortage of necessaries, according to (Depoux et al., 2020). Thus it is necessary to identify the right information from the social media text.

\section{Dataset}

The dataset provided by Nguyen et al. (2020) contains 10,000 English Tweets about COVID-19, which is used to automatically identify whether a tweet contains useful information about the COVID-19 (informative) or not (uninformative). There are 4,719 INFORMATIVE tweets and 5,281 UNINFORMATIVE tweets in the dataset, and three different annotators annotate each tweet. The inter-annotator agreement calculated by Fleiss' Kappa score of the dataset is $81.80 \%$. Also, the dataset is split into the training, development, and test sets with proportion 7-1-2. Table 2 shows the overview information about the dataset.

\begin{tabular}{lrr}
\hline & INFORMATIVE & UNINFORMATIVE \\
\hline Training & 3,303 & 3,697 \\
Development & 472 & 528 \\
Test & 944 & 1,056 \\
\hline
\end{tabular}

Table 2: Overview of the WNUT-2020 Task 2 dataset.

\section{Methodologies}

In this paper, we propose an ensemble method that combines the deep learning models with the transfer learning models to identify information about COVID-19 from users' tweets.

\subsection{Deep neural model}

We implement the Bi-GRU-CNN model, which was used for salary prediction by Wang et al. (2019) and Job prediction by Van Huynh et al. (2020), with the GloVe-300d word embedding (Pennington et al., 2014). This model consists of three main layers: the word representation layers (word embedding), the 1D Convolutional layers (CONV-1D), and the bidirectional GRU layer (Bi-GRU). This model also achieved high performances on previous study works(Wang et al., 2019; Van Huynh et al., 2019, 2020). Fig 1 illustrates the Bi-GRUCNN model.

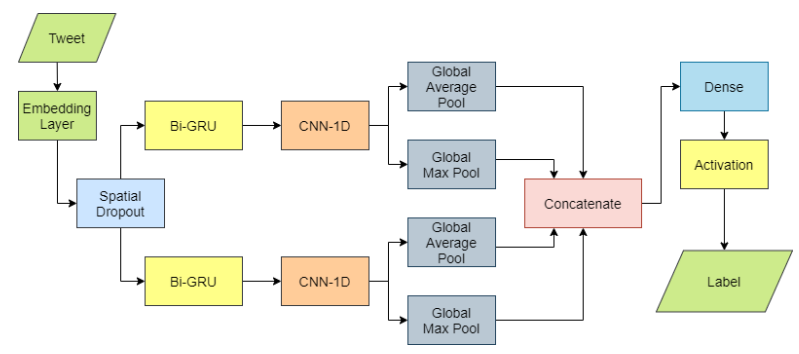

Figure 1: Overview architecture of Bi-GRU-CNN model.

\subsection{Transfer learning model}

Inspired by transfer learning success on many NLP tasks such as text classification (Do and Ng, 2006; Rizoiu et al., 2019) and machine reading comprehension (Devlin et al., 2019; Van Nguyen et al., 2020). In this paper, we used the SOTA transfer learning models, such as BERT (Devlin et al., 2019), RoBERTa (Liu et al., 2019), and XLNet (Yang et al., 2019) with fine-tuning techniques for the problem of identifying informative tweet about COVID-19. In our experiment, we used the pretrained language model, as described in Table 3. All of these pre-trained models are constructed on English texts. 


\begin{tabular}{ll}
\hline Transfer model & Pre-trained model \\
\hline BERT & bert_en_uncased \\
RoBERTa & roberta-base \\
XLNet & xlnet-large-cased \\
\hline
\end{tabular}

Table 3: List of transformer models used in our experiment.

\subsection{Ensemble method}

As the success of the ensemble models of previous tasks (Van Huynh et al., 2020; Nguyen et al., 2019), we propose a simple yet effective ensemble approach with the majority voting between the outputs of four different models, including Bi-GRUCNN, BERT, RoBERTa, and XLNet for classifying whether a tweet contains information about COVID-19 or not. Fig 2 describes our ensemble model.

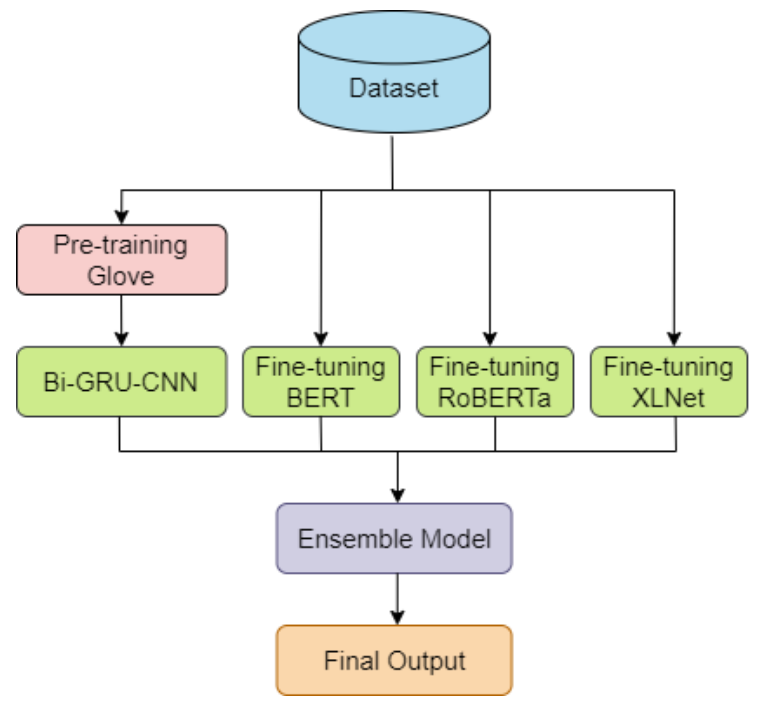

Figure 2: Overview architecture of our ensemble approach.

\section{Experiment}

\subsection{Experimental settings}

In this study, we experimented with datasets provided by WNUT-2020 Task 2 . Training, development, and testing sets are divided as described in Section 3. To evaluate our models, we use four metrics include accuracy, precision, recall, and F1.

To prepare data for the model training and model evaluation phases, we perform the simple and effective pre-processing of input data as follows:

- Step 1: Converting the tweet into the lowercase strings.
- Step 2: Removing the user names in the tweets.

- Step 3: Deleting all URLs in the tweets.

- Step 4: Representing words into vectors with pre-trained word embedding sets for deep neural network models.

According to analyzing the length of the tweets in the data, we set max_length of the models to be 512 and epochs to be 15 for two models $\mathrm{Bi}$ GRU-CNN and XLNet, and 3 for model BERT and RoBERTa. After searching for extensive hyperparameter, we set learning_rate equal to $1 \mathrm{e}-3$ and dropout equal to 0.2 for the Bi-GRU-CNN model and learning_rate equal to $1 \mathrm{e}-5$ and dropout equal to 0.1 for three models BERT, RoBERTa, and XLNet.

\subsection{Experimental results}

Experimental results of the single model and the ensemble model on the development set are presented in Table 4. Specifically, in the single models, the Bi-GRU-CNN model gives the lowest performance with $85.66 \%$ by $\mathrm{F} 1$ and $86.10 \%$ by accuracy. The single model with the highest efficiency is XLNet, which attained $89.86 \%$ by $\mathrm{F} 1$ and $90.30 \%$ by accuracy. In addition, the BERT model gives the highest Precision with $89.53 \%$, and the RoBERTa model achieved the highest Recall result with $90.74 \%$. In particular, our recommend ensemble model gives the best performance when combining the power of single models together, which accomplished $90.65 \%, 91.00 \%$, and $92.37 \%$ by F1, Accuracy, and Recall respectively, according to Table 4. Specifically, our model improved $0.79 \%$ by F1 and $0.70 \%$ by Accuracy over the most extensive single model (XLNet), and $0.63 \%$ by Recall over the RoBERTa model.

\begin{tabular}{lrrrc}
\hline \multicolumn{1}{c}{ Model } & Accuracy & Precision & Recall & F1 \\
\hline Bi-GRU-CNN & 86.10 & 83.50 & 87.92 & 85.66 \\
BERT & 89.79 & $\mathbf{8 9 . 5 3}$ & 88.77 & 89.15 \\
RoBERTa & 89.90 & 87.47 & $\mathbf{9 1 . 7 4}$ & 89.56 \\
XLNet & $\mathbf{9 0 . 3 0}$ & 88.66 & 91.10 & $\mathbf{8 9 . 8 6}$ \\
Ensemble & $\mathbf{9 1 . 0 0}$ & $\mathbf{8 8 . 9 8}$ & $\mathbf{9 2 . 3 7}$ & $\mathbf{9 0 . 6 5}$ \\
\hline
\end{tabular}

Table 4: Model performances on the development set of the COVID-19 dataset

After the system evaluation of WNUT-2020 Task 2 , Table 5 displays our ensemble model results on the testing set. This result is compared with the top 5 highest teams' results and the baseline model 
(BASELINE - FASTTEXT). Our model with F1 is $88.81 \%, 2.15 \%$ lower than the first rank team, and $13.78 \%$ higher than the baseline model. As for the results of accuracy, we get $89.40 \%, 2.10 \%$ lower than the first rank team, and $12.10 \%$ higher than the baseline model.

\begin{tabular}{lrrrc}
\hline \multicolumn{1}{c}{ Team Name } & Accuracy & Precision & Recall & F1 \\
\hline NutCracker & 91.50 & 91.35 & 90.57 & 90.96 \\
NLP_North & 91.40 & 90.29 & 91.63 & 90.96 \\
SupportNUTMachine & 91.40 & 90.46 & 91.42 & 90.94 \\
\#GCDH & 91.25 & 89.19 & 92.69 & 90.91 \\
Loner & 91.20 & 89.18 & 92.58 & 90.85 \\
BASELINE - FASTTEXT & 77.30 & 72.88 & 77.10 & 75.03 \\
\hline BANANA & $\mathbf{8 9 . 4 0}$ & $\mathbf{8 8 . 5 3}$ & $\mathbf{8 9 . 0 9}$ & $\mathbf{8 8 . 8 1}$
\end{tabular}

Table 5: Comparison with our model's performance with that of other teams.

\subsection{Result analysis}

Fig. 3 describes the confusion matrix of the ensemble methods when predicting informative tweets about COVID-19. It can be inferred from Fig. 3 that the ability of prediction correct label on the INFORMATIVE label is higher than the UNINFORMATIVE label.

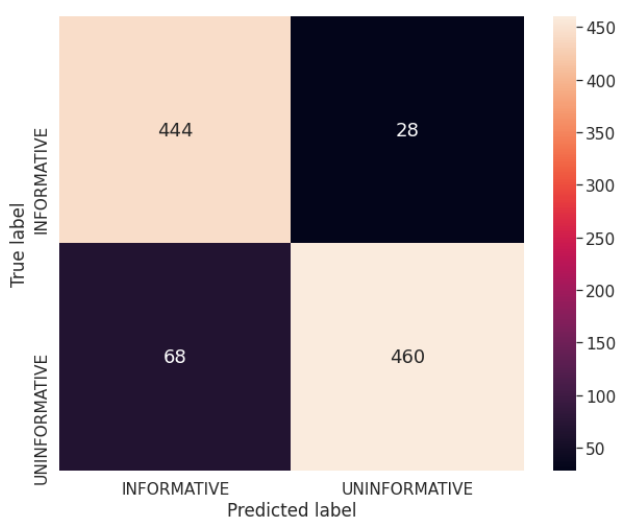

Figure 3: Confusion matrix of our ensemble model on the COVID English Tweet dataset.

In addition, Table 6 displays some error prediction examples from the dataset. Most of the wrong predictions occurred because of the appearance of special characters such as hashtag, the HTTPURL phrases, which stand for the URL links in the tweets. For the INFORMATION tweets, the appearance of the HTTPURL phrase and the hashtag \#coronavirus make the classification model predict the wrong label. This mistake is the same for the UNINFORMATION tweets, where the appearance of HTTPURL phrase and the hashtags related to the Coronavirus affected the results of the prediction model.

\begin{tabular}{lll}
\hline Tweet & PL & TL \\
\hline NATIONAL NEWS: Coronavirus: Linda & UN & IN \\
Lusardi says COVID-19 made her want to & & \\
die and turned her vomit blue HTTPURL & & \\
\hline Oh, he was sick before getting on the flight & UN & IN \\
back to Australia. So Vail, Denver and pos- & & \\
sibly LA (if the layover was long enough) & & \\
are suspect. I bet the LA airport is where & & \\
he got it :( \#coronavirus & & \\
\hline 20/03/20 PRESS RELEASE Council Urges & UN & IN \\
Consumers to be Considerate Fears about a & & \\
positive coronavirus (COVID-19) case led & & \\
shoppers in Fiji to begin stocking up on & & \\
supplies to fill pantries. HTTPURL & &
\end{tabular}

(a) Misclassified INFORMATION examples.

\begin{tabular}{|c|c|c|}
\hline Tweet & PL & TL \\
\hline $\begin{array}{l}\text { Vegetable market Peshawar KP.! People } \\
\text { here are least worried about \#COVID19 } \\
. ! 1 \text { infected Asymptomatic person and he } \\
\text { would be transmitting it to complete city } \\
\text { inside and outside! HTTPURL }\end{array}$ & IN & UN \\
\hline $\begin{array}{l}\text { FYI, the state's \#COVID19 stats show } 395 \\
\text { people have been tested via state lab. But } \\
\text { doesn't show a total for all labs. HTTPURL }\end{array}$ & IN & $\overline{\mathrm{UN}}$ \\
\hline $\begin{array}{l}\text { New post (Mike Pence Celebrates Story of } \\
\text { Great Great Grandmother Recovering from } \\
\text { Coronavirus) has been published on Randy } \\
\text { Salars News And Comment - HTTPURL } \\
\text { HTTPURL }\end{array}$ & IN & UN \\
\hline
\end{tabular}

(b) Misclassified UNINFORMATION examples.

Table 6: Misclassified examples. PL: predicted label, TL: true label

\section{Conclusion and future work}

This paper has addressed our work on the WNUT2020 Task 2: Identifying COVID-19 Information on Twitter. We proposed our ensemble model combining the deep learning models and the transfer learning models for detecting information about COVID-19 from users' tweets. Our ensemble model achieved $91.00 \%$ by accuracy and $90.65 \%$ by F1 on the development set, and achieved $89.04 \%$ by accuracy and $88.81 \%$ by $\mathrm{F} 1$ on the public test set, which ranked \#25 in the competition.

In the future, we will improve our model's performance by exploring different features of the users' tweets and transfer learning models with fine-tuning techniques. Finally, we hope our study can be applied in practice for detecting COVID19 from social networks to support the COVID-19 battle all over the world.

\section{References}

Emily Chen, Kristina Lerman, and Emilio Ferrara. 2020. Tracking social media discourse about the 
covid-19 pandemic: Development of a public coronavirus twitter data set. JMIR Public Health and Surveillance, 6(2):e19273.

Anneliese Depoux, Sam Martin, Emilie Karafillakis, Raman Preet, Annelies Wilder-Smith, and Heidi Larson. 2020. The pandemic of social media panic travels faster than the COVID-19 outbreak. Journal of Travel Medicine, 27(3). Taaa031.

Jacob Devlin, Ming-Wei Chang, Kenton Lee, and Kristina Toutanova. 2019. BERT: Pre-training of deep bidirectional transformers for language understanding. In Proceedings of the 2019 Conference of the North American Chapter of the Association for Computational Linguistics: Human Language Technologies, Volume 1 (Long and Short Papers), pages 4171-4186, Minneapolis, Minnesota. Association for Computational Linguistics.

Chuong B Do and Andrew Y Ng. 2006. Transfer learning for text classification. In Advances in Neural Information Processing Systems, pages 299-306.

Ensheng Dong, Hongru Du, and Lauren Gardner. 2020. An interactive web-based dashboard to track covid19 in real time. The Lancet Infectious Diseases, 20.

Bennett Kleinberg, Isabelle van der Vegt, and Maximilian Mozes. 2020. Measuring emotions in the covid-19 real world worry dataset. arXiv preprint arXiv:2004.04225.

Rabindra Lamsal. 2020. Coronavirus (covid-19) tweets dataset.

Yinhan Liu, Myle Ott, Naman Goyal, Jingfei Du, Mandar Joshi, Danqi Chen, Omer Levy, Mike Lewis, Luke Zettlemoyer, and Veselin Stoyanov. 2019. Roberta: A robustly optimized bert pretraining approach.

Dat Quoc Nguyen, Thanh Vu, Afshin Rahimi, Mai Hoang Dao, Linh The Nguyen, and Long Doan. 2020. WNUT-2020 Task 2: Identification of Informative COVID-19 English Tweets. In Proceedings of the 6th Workshop on Noisy User-generated Text.

Duc-Vu Nguyen, Kiet Van Nguyen, and Ngan LuuThuy Nguyen. 2019. Nlp@uit at vlsp 2019: A simple ensemble model for vietnamese dependency parsing. The Sixth International Workshop on Vietnamese Language and Speech Processing VLSP 2019.

Jeffrey Pennington, Richard Socher, and Christopher D. Manning. 2014. Glove: Global vectors for word representation. In Empirical Methods in Natural Language Processing (EMNLP), pages 1532-1543.

Marian-Andrei Rizoiu, Tianyu Wang, Gabriela Ferraro, and Hanna Suominen. 2019. Transfer learning for hate speech detection in social media. arXiv preprint arXiv:1906.03829.
Gautam Kishore Shahi, Anne Dirkson, and Tim A. Majchrzak. 2020. An exploratory study of covid-19 misinformation on twitter.

Tin Van Huynh, Vu Duc Nguyen, Kiet Van Nguyen, Ngan Luu-Thuy Nguyen, and Anh Gia-Tuan Nguyen. 2019. Hate speech detection on vietnamese social media text using the bi-gru-lstm-cnn model. arXiv preprint arXiv:1911.03644.

Tin Van Huynh, Kiet Van Nguyen, Ngan Luu-Thuy Nguyen, and Anh Gia-Tuan Nguyen. 2020. Job prediction: From deep neural network models to applications. In 2020 RIVF International Conference on Computing and Communication Technologies (RIVF), pages 1-6. IEEE.

Kiet Van Nguyen, Duc-Vu Nguyen, Anh Gia-Tuan Nguyen, and Ngan Luu-Thuy Nguyen. 2020. New vietnamese corpus for machine reading comprehension of health news articles. arXiv preprint arXiv:2006.11138.

Zhongsheng Wang, Shinsuke Sugaya, and Dat PT Nguyen. 2019. Salary prediction using bidirectionalgru-cnn model. Assoc. Nat. Lang. Process.

Zhilin Yang, Zihang Dai, Yiming Yang, Jaime Carbonell, Russ R Salakhutdinov, and Quoc V Le. 2019. Xlnet: Generalized autoregressive pretraining for language understanding. In Advances in Neural Information Processing Systems 32, pages 5753-5763. Curran Associates, Inc. 